\title{
All-optical runaway evaporation to Bose-Einstein condensation
}

\author{
J.-F. Clément, J.-P. Brantut, M. Robert-de-Saint-Vincent, R. A. Nyman, * A. Aspect, T. Bourdel, and P. Bouyer \\ Laboratoire Charles Fabry de l'Institut d'Optique, CNRS, Université Paris Sud, Campus Polytechnique,
}

RD128, 91127 Palaiseau, France

(Received 12 March 2009; published 19 June 2009)

\begin{abstract}
We demonstrate runaway evaporative cooling directly with a tightly confining optical-dipole trap and achieve fast production of condensates of $1.5 \times 10^{5}{ }^{87} \mathrm{Rb}$ atoms. Our scheme uses a misaligned crossed-beam far off-resonance optical-dipole trap (MACRO-FORT). It is characterized by independent control of the trap confinement and depth allowing forced all-optical evaporation in the runaway regime. Although our configuration is particularly well suited to the case of ${ }^{87} \mathrm{Rb}$ atoms in a $1565 \mathrm{~nm}$ optical trap, where an efficient initial loading is possible, our scheme is general and will allow all-optical evaporative cooling at constant stiffness for every optically trappable atomic or even molecular species.
\end{abstract}

DOI: 10.1103/PhysRevA.79.061406

PACS number(s): 37.10.De, 05.30.Jp, 37.10.Gh, 32.60.+i

Far off-resonance optical-dipole traps (FORT) [1] are used extensively in ultracold atom experiments. They allow great versatility of the trapping potentials and therefore offer the possibility to study numerous physical situations such as double wells [2], two-dimensional (2D) traps [3], or artificial crystals of light [4]. Moreover, they have the advantage over magnetic traps that they leave the magnetic field as a degree of freedom. As a consequence, they are crucial to the study of Bose-Einstein condensates (BEC) with internal spin degrees of freedom [5] or formation of ultracold molecules by means of magnetically tuned Feshbach resonances [6-8]. Last and not least, for some atomic species, condensation in a magnetic trap is not possible and all-optical trapping and cooling is necessary. This is the case for cesium because of a large inelastic collision rate [9] and also for magnetically untrappable atoms such as ytterbium [10] or alkaline-earth atoms.

All-optical cooling methods to achieve quantum degeneracy have been successfully implemented in ultracold atom experiments, both for bosonic [11] and fermionic species [12]. These methods have several advantages. The absence of a magnetic trap permits a better optical access to the trapped cloud and a better control of residual magnetic field for precision measurements. The optical trap tight confinement allows fast evaporation ramps and therefore cycling time of only a few seconds. However, all-optical evaporative cooling is severely hindered by the fact that the trap confinement is reduced during forced evaporative cooling. This is because the reduction of the trap depth is usually performed by lowering the trap power (typically by several orders of magnitude), which consequently reduces the trap frequencies, the collision rate, and therefore also the evaporation efficiency. In early experiments, it was thus necessary to start evaporative cooling with a large phase-space density and a large collision rate [11]. Many elaborated strategies have then been used to improve the conditions for evaporative cooling in an optical trap: Raman sideband cooling in a three-dimensional (3D) lattice to lower the temperature be-

\footnotetext{
*Present address: Center for Cold Matter, Imperial College, London SW7 2BW, UK.
}

fore the transfer into the optical trap [13], a mobile lens to change the optical trap waist dynamically $[13,14]$, or the use of a second dipole trap to compress the cloud after some evaporation [9].

In this Rapid Communication, we present an all-optical method allowing one to decouple the control of the trap depth from the control of the trap confinement, in analogy to the case of radio-frequency forced evaporation in a magnetic trap. We are thus able to reach the runaway regime, where the collision rate increases during the evaporation, a situation which seems impossible to achieve in a single-beam optical trap [15]. Recently, a similar result has been obtained in the case of cesium atoms using an optical-dipole trap combined with a magnetic-field gradient [16]. In contrast to this hybrid scheme, our all-optical evaporation scheme does not rely on an external magnetic field and therefore does not require any specific magnetic properties. It is very general: it can be used to cool a spin mixture, as shown in this Rapid Communication, but also nonparamagnetic atoms, atomic mixtures, or even molecules.

Our misaligned crossed-beam far off-resonance dipole trap (MACRO-FORT) is composed of two horizontal crossing beams, a wide beam and a tightly confining beam, with waists of $180 \mu \mathrm{m}$ and $26 \mu \mathrm{m}$, respectively [Fig. 1(a)]. The tightly confining beam is offset by $\sim 80 \mu \mathrm{m}$ from the center of the wide beam as sketched in Fig. 1. It is responsible for most of the trap confinement and the atoms are trapped at its waist. On the contrary, the wide beam is used to control the trap depth $U_{\text {trap. }}$ It applies locally a force, which pulls the atoms out of the confining beam. By varying its power we can vary the trap depth and it acts as an effective evaporative knife which removes the high energy atoms from the trap. During the evaporation, we can thus control the trap depth and confinement independently. This method allows us to implement an efficient forced evaporation in the runaway regime to Bose-Einstein condensation.

In addition, our optical trap geometry is well suited to an efficient loading from the magneto-optical trap. This is done in two steps. We first combine the wide optical beam with an extremely far-detuned optical molasses. It takes advantage of a position selective optical pumping due to the strong light shifts induced by the wide trapping beam at $1565 \mathrm{~nm}$ [17]. Second, the atoms are transferred into the tightly confining 


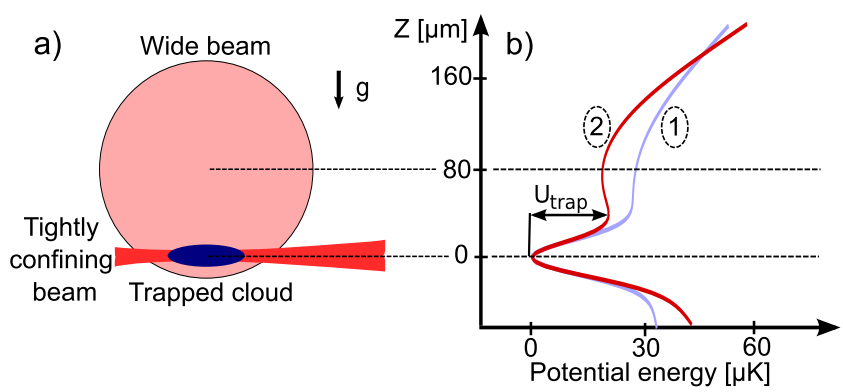

FIG. 1. (Color online) (a) Scheme of our MACRO-FORT. The confining beam crosses the wide beam at a distance of about $80 \mu \mathrm{m}$ from its center. This configuration decouples the control of the trap depth $U_{\text {trap }}$ from the control of the trap confinement. (b) Vertical potential energy cuts. The two curves correspond to the same power of $0.15 \mathrm{~W}$ in the confining beam whereas the wide beam powers are $8 \mathrm{~W}$ (light/blue curve 1) and $16 \mathrm{~W}$ (dark/red curve 2). It shows that the trap depth is reduced by increasing the wide beam power. The atoms, pulled upward from the confining beam waist, are lost in the direction of the wide beam, perpendicular to the figure, along which there is no confinement.

beam creating the trap configuration used for the evaporation.

The experimental sequence begins with a standard 3Dmagneto-optical trap (MOT) with $\sim 3 \times 10^{9}$ atoms, loaded from a $2 \mathrm{D}-\mathrm{MOT}$ in a few seconds, as described in [17]. The detuning of the cooling beams is then increased to $120 \mathrm{MHz}$ for $60 \mathrm{~ms}$, which leads to a compression of the cloud as the repelling forces due to multiple scattering are reduced [18]. At this point starts our dipole trap loading procedure. The wide trapping beam at $1565 \mathrm{~nm}$ with a waist of $180 \mu \mathrm{m}$ and a power of $28 \mathrm{~W}$ is turned on. Simultaneously the magneticfield gradient is turned off and the cooling laser detuning is increased to $200 \mathrm{MHz}$. This value is chosen such that the cooling beam remains red detuned even in the presence of the strong light shift induced by the trapping laser [Fig. 2(a)]. This corresponds to a very far-detuned optical molasses with the dipole trap beam intersecting the atomic cloud in its center. It lasts for $50 \mathrm{~ms}$.

Our dipole trapping beam not only affects the cooling transition but also detunes the repumping beam out of resonance, as shown in Fig. 2(a). The repumping efficiency decreases as the FORT laser intensity increases [Fig. 2(b)] and the atoms are pumped to the $F=1$ hyperfine states. This reduces scattered photon reabsorption, a process which limits the density of laser-cooled samples. We thus create an effective spatial dark MOT [19] induced by the trapping laser itself $[11,20]$. The depumping effect is further enhanced by reducing the repumping laser intensity by a factor $\sim 30$ to $21 \mu \mathrm{W} \mathrm{cm}{ }^{-2}$. In absorption imaging without repumping, we find that $99 \%$ of the atoms remaining in the dipole trap are in the $5 S_{1 / 2}(F=1)$ hyperfine states. For the untrapped atoms this number is only $97 \%$ showing that the dipole trapping laser causes an additional reduction in the repumping rate.

The cooling and repumping beams are then switched off and about $3 \times 10^{7}$ atoms remain in the wide trapping beam. Their temperature is $20 \mu \mathrm{K}$ and the phase-space density is $\sim 2 \times 10^{-5}$. Longitudinally they occupy a region of about 1 $\mathrm{mm}$ in size, but the trapping force in this direction is negli-

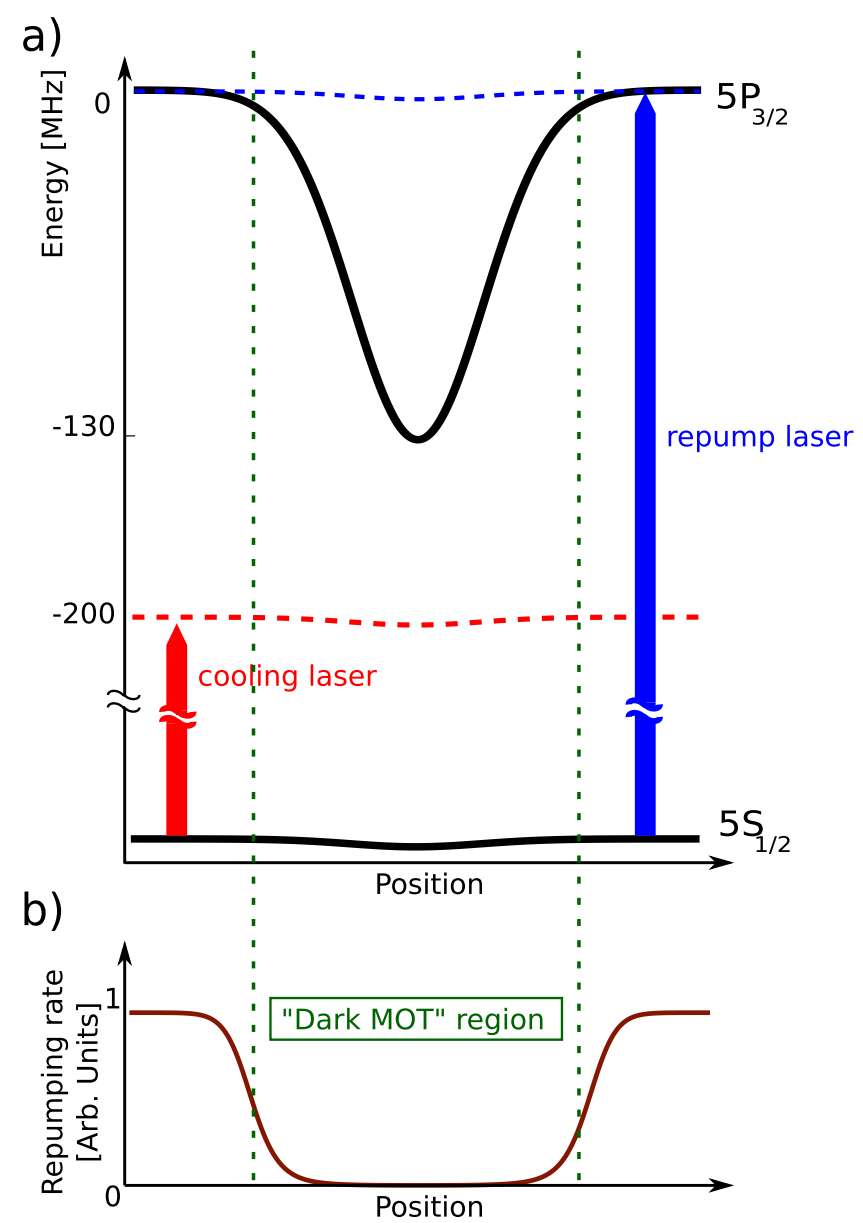

FIG. 2. (Color online) Loading scheme into the wide trapping beam. (a) Light shifted levels of the $5 S_{1 / 2}$ and $5 P_{3 / 2}$ states of ${ }^{87} \mathrm{Rb}$ under the influence of the wide beam at a power of $28 \mathrm{~W}$. At 1565 $\mathrm{nm}$, the $5 P_{3 / 2}$ excited state of the MOT cooling transition is 42.6 times more shifted than the $5 S_{1 / 2}$ fundamental state [17]. For clarity the hyperfine structure is not shown. During the very far-detuned molasses phase, the cooling laser is $200 \mathrm{MHz}$ detuned and therefore remains to the red of the cycling transition even in the presence of the trapping laser. The repumping light on the contrary is on resonance in free space and is brought out of resonance by the dipole laser light. (b) Repumping rate as a function of position. The repumping is not efficient at the position of the dipole trap leading to a depumping effect leaving the atom into the "dark" $F=1$ hyperfine states.

gible and the atoms can escape along the beam. In order to create a trap in three dimensions, we add the tightly confining beam immediately after switching off the molasses. It intersects the wide beam at an angle of $56^{\circ}$. We choose a quite small waist of $26 \mu \mathrm{m}$ and a power of $6 \mathrm{~W}$ [21] in order to create a tight trap, whose frequencies are $124 \mathrm{~Hz}, 3.8 \mathrm{kHz}$, and $3.8 \mathrm{kHz}$. After a waiting time of $100 \mathrm{~ms}$, we end up with $3 \times 10^{6}$ atoms at $65 \mu \mathrm{K}$ whereas the rest of the atoms are lost. The phase-space density is $2.5 \times 10^{-3}$. Adding the tightly confining beam has thus allowed us to increase the phase-space density by 2 orders of magnitude, with only a factor of 10 reduction in atom number. We attribute this to an efficient free-evaporative cooling.

This result is nonintuitive as the initial collision rate in the 


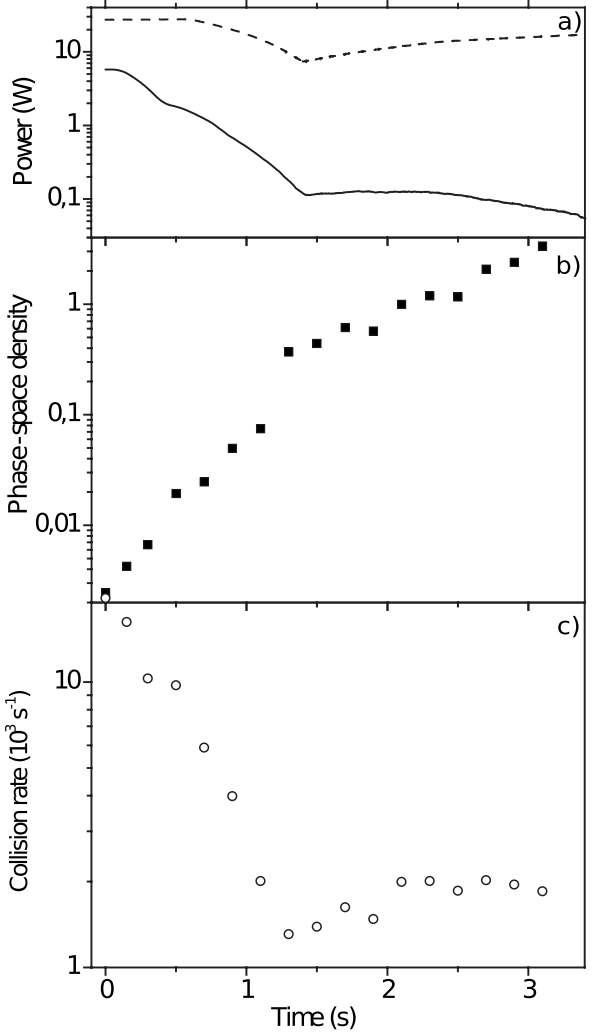

FIG. 3. (a) Optical power in the two trapping beams as a function of time during the evaporation. The solid (dashed) curve corresponds to the tightly confining (wide) beam. In the second part of the evaporation the tightly confining beam power is roughly constant whereas the power of the wide beam is increased to force the evaporation. [(b) and (c)] Phase-space density and collision rate as a function of time.

wide beam is a priori too low to prevent atoms which fall into the tightly confining beam to escape from it. However, a one-body calculation using our trapping potential showed that these atoms follow nontrivial 3D trajectories and typically remain for a time much longer than the $100 \mathrm{~ms}$ waiting time in the crossed-trap region where they accumulate. As a consequence, the atomic density and the collision rate quickly increase in the tightly confining trap. The atoms then thermalize and cool down by evaporation. The final collision rate is greater than $10^{4} \mathrm{~s}^{-1}$ and the conditions are very favorable for efficient evaporative cooling.

We now proceed to the final forced evaporative cooling stage. It takes advantage of our specific MACRO-FORT geometry, which permits independent control of trap stiffness and depth. The collision rate and the phase-space density during the evaporation are presented in Fig. 3. The time sequence is the result of an optimization of the number of atoms obtained in the condensate. The evaporation can be decomposed in two phases. In a first step (which lasts $1.4 \mathrm{~s}$ ), we decrease the power of both beams. Evaporative cooling is then accompanied with a reduction in the confinement, the density, and the collision rate. Such a reduction is useful as it avoids three-body losses [22]. In a second step (which lasts 2 s) we increase the power of the wide beam while the tightly confining beam is approximately kept at a constant power. As explained in the introduction, because of our original trap geometry, this procedure yields forced evaporation at constant confinement (Fig. 1).

Runaway evaporation is experimentally observed as the collision rate increases during this phase before it saturates due to three-body losses [Fig. 3(b)]. Our evaporation ramp, which lasts $3 \mathrm{~s}$, leads to $3 \times 10^{5}$ atoms at the critical temperature and to pure condensates of $1.5 \times 10^{5}$ atoms. However, by simply reducing the duration of all ramps we were able to achieve Bose-Einstein condensation in an evaporation as short as $650 \mathrm{~ms}$. Our condensates are spinor condensates with relative abundance of $0.45,0.35$, and 0.2 in the $m_{F}=$ $-1, m_{F}=0$, and $m_{F}=1$ magnetic states of the $F=1$ manifold. In order to produce a BEC in a single state, a spin distillation method [20] could be used.

We characterize the efficiency of our evaporation ramp through the scaling parameter $\gamma=-\frac{\mathrm{d} \operatorname{Ln} D}{\mathrm{~d} \operatorname{Ln} N}=2.8(5)$, where $D$ is the phase-space density and $N$ is the number of atoms. Given our estimated truncation parameter $\eta=11(2)$ (ratio of trap depth to temperature), the efficiency is lower than expected according to the scaling laws [15]. The reduction can be understood by including three-body losses in the analysis. Using a similar treatment to [23,24], the efficiency of the evaporation is given by

$$
\gamma=(\eta-4)-R(\eta-2)
$$

The term $\eta-4$ comes from the evaporation [15] while the second term is due to losses; $R$ is the ratio of three-body losses to the total number of atoms removed from the trap [25]. With $\eta=11(2)$, we recover our observed efficiency of the evaporation with $R \approx 0.45$. Such a number is compatible with our calculated three-body loss rate of about $1 \mathrm{~s}^{-1}$ at the end of the evaporation.

In conclusion, we have demonstrated an efficient and simple all-optical route to Bose-Einstein condensation of ${ }^{87} \mathrm{Rb}$ in a $1565 \mathrm{~nm}$ dipole trap [26]. A very far off-resonance optical molasses, taking advantage of the strong light shifts, is used to efficiently load the optical-dipole trap. Then we apply a misaligned crossed-beam configuration (MACROFORT), which permits all-optical runaway evaporation. This method can be straightforwardly generalized to numerous situations such as the cooling of nonparamagnetic atoms, spin mixtures, atomic mixtures, or molecules. The ability to achieve high duty cycle without a magnetic trap opens the perspective to use BECs in high precision measurement applications such as atomic clocks or accelerometers [27].

We acknowledge G. Varoquaux for his help in the construction of the experimental apparatus, F. Moron and A. Villing for technical assistance, G. Lucas-Leclin, D. GuéryOdelin, and J. V. Porto for helpful discussions, and ImagineOptics company for lending us a HASO-NIR wave-front analyzer. This research was supported by CNRS, CNES as part of the ICE project, the project "blanc" MélaBoFérIA from ANR, IFRAF, QUDEDIS, by the STREP program FINAQS of the European Union, and by the MAP program SAI of the European Space Agency (ESA). 
[1] J. D. Miller, R. A. Cline, and D. J. Heinzen, Phys. Rev. A 47, R4567 (1993).

[2] J. Esteve, C. Gross, A. Weller, S. Giovanazzi, and M. K. Oberthaler, Nature (London) 455, 1216 (2008).

[3] Z. Hadzibabic, P. Krüger, M. Cheneau, B. Battelier, and J. Dalibard, Nature (London) 441, 1118 (2006).

[4] M. Greiner, O. Mandel, T. Esslinger, T. W. Hänsch, and I. Bloch, Nature (London) 415, 39 (2002).

[5] J. Stenger, S. Inouye, D. M. Stamper-Kurn, H.-J. Miesner, A. P. Chikkatur, and W. Ketterle, Nature (London) 396, 345 (1998).

[6] E. A. Donley, N. R. Claussen, S. T. Thompson, and C. E. Wieman, Nature (London) 417, 529 (2002).

[7] T. Bourdel, J. Cubizolles, L. Khaykovich, K. M. F. Magalhaes, S. J. J. M. F. Kokkelmans, G. V. Shlyapnikov, and C. Salomon, Phys. Rev. Lett. 91, 020402 (2003).

[8] C. A. Regal, C. Ticknor, J. L. Bohn, and D. S. Jin, Nature (London) 424, 47 (2003).

[9] T. Weber, J. Herbig, M. Mark, H.-C. Nägerl, and R. Grimm, Science 299, 232 (2003).

[10] Y. Takasu, K. Maki, K. Komori, T. Takano, K. Honda, M. Kumakura, T. Yabuzaki, and Y. Takahashi, Phys. Rev. Lett. 91, 040404 (2003).

[11] M. D. Barrett, J. A. Sauer, and M. S. Chapman, Phys. Rev. Lett. 87, 010404 (2001).

[12] S. R. Granade, M. E. Gehm, K. M. O'Hara, and J. E. Thomas, Phys. Rev. Lett. 88, 120405 (2002).

[13] T. Kinoshita, T. R. Wenger, and D. S. Weiss, Phys. Rev. A 71, 011602(R) (2005).

[14] Ming-Shien Chang, Ph.D. thesis, Georgia Institute of Technology, 2006 (http://www.physics.gatech.edu/ultracool/Papers).

[15] K. M. O'Hara, M. E. Gehm, S. R. Granade, and J. E. Thomas, Phys. Rev. A 64, 051403(R) (2001).

[16] C.-L. Hung, X. Zhang, N. Gemelke, and C. Chin, Phys. Rev. A
78, 011604(R) (2008).

[17] J. P. Brantut, J. F. Clément, M. Robert-de-Saint-Vincent, G. Varoquaux, R. A. Nyman, A. Aspect, T. Bourdel, and P. Bouyer, Phys. Rev. A 78, 031401(R) (2008).

[18] W. Petrich, M. H. Anderson, J. R. Ensher, and E. A. Cornell, J. Opt. Soc. Am. 11, 1332 (1994).

[19] W. Ketterle, K. B. Davis, M. A. Joffe, A. Martin, and D. E. Pritchard, Phys. Rev. Lett. 70, 2253 (1993).

[20] A. Couvert, M. Jeppesen, T. Kawalec, G. Reinaudi, R. Mathevet, and D. Guery-Odelin, Europhys. Lett. 83, 50001 (2008).

[21] Actually the tightly confining beam is already on with $\sim 0.7 \mathrm{~W}$ during the very far-detuned molasses phase. Such a power corresponds to a light shift similar to that created by the wide beam and the additional trapping volume permits a gain of $\sim 10 \%$ in the atom number.

[22] An experimental proof of the role of three-body collisions is the fact that using more power $(14 \mathrm{~W}$ rather than $6 \mathrm{~W})$ in the confining beam leads to a lower atom number.

[23] O. J. Luiten, M. W. Reynolds, and J. T. M. Walraven, Phys. Rev. A 53, 381 (1996).

[24] W. Ketterle and N. J. van Druten, Adv. At., Mol., Opt. Phys. 37, 181 (1996).

[25] For background losses, the formula is $\gamma=\eta-4-R(\eta-3)$ [24]. The difference comes from the fact that three-body losses mostly affect the atoms at the trap center where the density is higher.

[26] To further simplify the degenerated gas apparatus, all the laser beams for trapping and for cooling could be generated from a single $1560 \mathrm{~nm}$ fibered diode after amplification and frequency doubling [28].

[27] Y. Le Coq, J. A. Retter, S. Richard, A. Aspect, and P. Bouyer, Appl. Phys. B: Lasers Opt. 84, 627 (2006).

[28] R. Thompson, M. Tu, D. Aveline, N. Lundblad, and L. Maleki, Opt. Express 11, 1709 (2003). 